\title{
Mass spectrometric protein identification from two-dimensional gel separation with stain-free detection and visualization using native fluorescence
}

\author{
Iuliana Susnea a , Bogdan Bernevic a , Eliska Svobodova ${ }^{a}$, Diliana Dancheva Simeonovab, \\ Michael Wicke ${ }^{\mathrm{c}}$, Carsten Werner ${ }^{\mathrm{c}}$, Bernhard Schink ${ }^{\mathrm{b}}$, Michael Przybylski ${ }^{\mathrm{a}, *}$ \\ a Laboratory of Analytical Chemistry and Biopolymer Structure Analysis, Department of Chemistry, University of Konstanz, 78457 Konstanz, Germany \\ b Laboratory of Microbial Ecology, Department of Biology, University of Konstanz, 78457 Konstanz, Germany \\ c Institute of Animal Breeding and Genetics, University of Göttingen, 37075 Göttingen, Germany
}

Keywords:

Native fluorescence

Stain-free gel electrophoresis

Mass spectrometry

Protein identification

Porcine muscle proteomics

\begin{abstract}
A B S T R A C T
We describe here an approach for the mass spectrometric identification of proteins in proteome analysis from 1D- and 2D-gel electrophoretic separation, using stain-free detection and visualization based on native fluorescence. Staining procedures such as by Coomassie Brilliant Blue, silver salts and fluorescent dyes are typically employed to visualize gel-separated protein bands with high detection sensitivity, however all of these staining procedures produce significant background in mass spectrometric analysis. Using the native fluorescence of aromatic protein amino acids with UV transmission at $343 \mathrm{~nm}$ as a fast gel imaging system, unstained visualized protein spots were localised. Upon excision from gels using precise spot picking tools, gel spots were proteolytically digested and analysed by matrix-assisted laser desorption-ionisation mass spectrometry (MALDI-MS). After initial development and testing using 1Dgel separated standard proteins, the stain-free detection approach was successfully applied to MALDI-MS protein identifications in (i), bacterial proteomics of Desulfotignum phosphitoxidans, and (ii), in porcine skeleton muscle proteomics. Major advantages of the stain-free gel detection approach are (i), rapic analysis of proteins from 1D- and 2D-gel separations without destaining required before proteolytic digestion; (ii), low detection limits of proteins in gels; and (iii), low background in the mass spectrometric

analysis of proteins.
\end{abstract}

\section{Introduction}

A variety of protein detection and visualization techniques of protein bands or spots from 1- and 2-dimensional gel electrophoretic separations have been developed and employed in mass spectrometric proteomics [1-4]. Well established staining procedures for visualization of proteins in gels have used dyes such as Coomassie Brilliant Blue, silver salts and fluorescent dyes (Flamingo, Sypro ${ }^{\circledR}$ Ruby) $[5,6]$. While several of these approaches provide high detection sensitivities of proteins, major problems are frequently encountered with the compatibility of staining procedures with the mass spectrometric analysis [7], background arising

Abbreviations: BAGb, ioanalyzer gel; 2D, two-dimensional gel electrophoresis; IEF, isoelectric focusing; 1D, one-dimensional gel electrophoresis; SDS-PAGE sodium dodecyl sulfate polyacrylamide gel electrophoresis; MALDI-TOF, matrix assisted laser desorption/ionization - time of flight; PMF, peptide mass fingerprinting.

* Corresponding author. Tel.: +49 7531 882249; fax: +49 7531883097

E-mail address: Michael.Przybylski@uni-konstanz.de (M. Przybylski). from polar staining materials, and the need for applying destaining procedures of isolated proteins [8].

Several procedures have been recently explored in order to overcome these problems, using unstained gels in gel electrophoretic separations [9-12]. In fluorescence labeling studies of glycoproteins, Zhao and co-workers observed a fluorescent signal for non-glycosylated proteins such as hen lysozyme, which was attributed to the intrinsic (native) protein fluorescence [13]. Fluorescence detection of proteins has been evaluated with preor post-electrophoretic incorporation of halogenated compounds such as trichloromethane, trichloroethanol and trichloroacetic acid, which react with tryptophane residues upon treatment with UV light yielding products that show emission in the visible light range suitable for visualization of protein bands $[11,14]$. A direct UV fluorescence detection method for unstained proteins in gels was first developed by Roegener et al. who used laser excitation with $280 \mathrm{~nm}$ UV light $\left(35 \mathrm{~mJ} / \mathrm{cm}^{2}\right)$ and showed the visualization of proteins in both 1D- and 2D-gel separations with low detection limits (1-5 ng) [9]. A commercial gel bioanalyzer based on native fluorescence has been recently developed (LaVision-BioTec, Bielefeld, Germany) [15]. 
In the present study we have developed and applied native fluorescence detection of proteins in stain-free one- and twodimensional gel electrophoretic separations as a sensitive and efficient approach for mass spectrometric identifications in proteome analysis. We show that in stain-free visualization the protein fixation step following gel electrophoretic separation can be omitted, and protein spots or bands directly analysed after scanning. Successful applications using the stain-free gel bioanalyzer were performed in bacterial proteome studies (Desulfotignum phosphitoxidans) $[16,17]$, and in porcine skeleton muscle proteomics. These results, providing unequivocal identifications of proteins at low detection limits, without the need for destaining procedures before proteolytic digestion of isolated protein bands, indicate broad applicability of the stain-free gel separation and visualization approach as an efficient and sensitive tool in mass spectrometric proteomics.

\section{Experimental part}

\subsection{D- and 2D-gel electrophoresis}

Proteins were separated by $12 \%$ sodium dodecyl sulfate polyacrylamide gel electrophoresis (SDS-PAGE) on $1 \mathrm{~mm}$ gels using the standard Laemmli method with a Mini-PROTEAN ${ }^{\circledR} 3$ cell gel system (Bio-Rad; München, Germany). Hen eggwhite lysozyme and other model proteins were obtained from Sigma-Aldrich (München, Germany).

Samples for 2D-gel separations were applied for $12 \mathrm{~h}$ on $17 \mathrm{~cm}$ IPG strips (pH ranges, 5-8 and 3-10) using a passive in-gel rehydration method. The rehydration solution contained $7 \mathrm{M}$ urea, $2 \mathrm{M}$ thiourea, $4 \%(\mathrm{w} / \mathrm{v})$ CHAPS, $40 \mathrm{mM}$ Tris-base, $2 \%(\mathrm{v} / \mathrm{v})$ Servalyt 5-8, $0.3 \%$ dithiothreitol (DTT) and a trace of bromophenol blue. Isoelectric focusing (IEF) was carried out using a Multiphor horizontal electrophoresis system (Amersham Biosciences, München, Germany). After focusing, the IPG strips were equilibrated for $30 \mathrm{~min}$ in $6 \mathrm{M}$ urea, $30 \%$ glycerol, $2 \%(\mathrm{w} / \mathrm{v}) \mathrm{SDS}, 0.05 \mathrm{M}$ Tris $-\mathrm{HCl}(\mathrm{pH} 8.8)$, $1 \%(\mathrm{w} / \mathrm{v})$ DTT and a trace of bromophenol blue, then incubated for $30 \mathrm{~min}$ in the same solution except that DTT was replaced by $4.5 \%(\mathrm{w} / \mathrm{v})$ iodoacetamide. For the second separation step, the BioRad Protean-II-xi vertical electrophoresis system was, and $10 \%$ and $12.5 \%$ SDS-PAGE gels of $1.5 \mathrm{~mm}$ thickness were prepared. Strips were placed on the vertical gels and overlaid with $0.5 \%$ agarose in SDS running buffer ( $25 \mathrm{mM}$ Tris-base, $192 \mathrm{mM}$ glycine, $0.1 \%(\mathrm{w} / \mathrm{v})$ SDS). Electrophoresis was performed in two steps: $25 \mathrm{~mA} / \mathrm{gel}$ for approximately $30 \mathrm{~min}$, and $40 \mathrm{~mA} / \mathrm{gel}$ until the dye front reached the anodic end of the gels.

For the 2D-gel preparations of samples from D. phosphitoxidans (soluble fraction), acetone precipitation was used for removal of salts and contaminants. The protein fraction was precipitated for $5 \mathrm{~h}$ at $-28 \mathrm{C}$ by adding 6 volumes of ice-cold acetone. After $20 \mathrm{~min}$ centrifugation at $14900 \times \mathrm{g}$ the residual acetone was removed and the pellet allowed to dry.

\subsection{Native fluorescence visualization of proteins}

Following 1D- or 2D-gel separations, proteins were visualized using sensitive colloidal Coomassie staining and silver staining as described $[5,6]$ using a GS-710 calibrated imaging densitometer (Bio-Rad, München, Germany), or scanned with the gel bioanalyzer (BAG). The components of the gel bioanalyzer (LaVision-Biotec $\mathrm{GmbH}$, Bielefeld, Germany) are schematically shown in Fig. 1. The experimental setup is based on a UV excitation source and a detection system within the UV range. The UV excitation light was generated by a $300 \mathrm{~W}$ xenon lamp $(265-680 \mathrm{~nm})$. The irradiation area was set to $1 \mathrm{~cm}^{2}$ at $35 \mathrm{~mW} / \mathrm{cm}^{2}$ and imaged by three

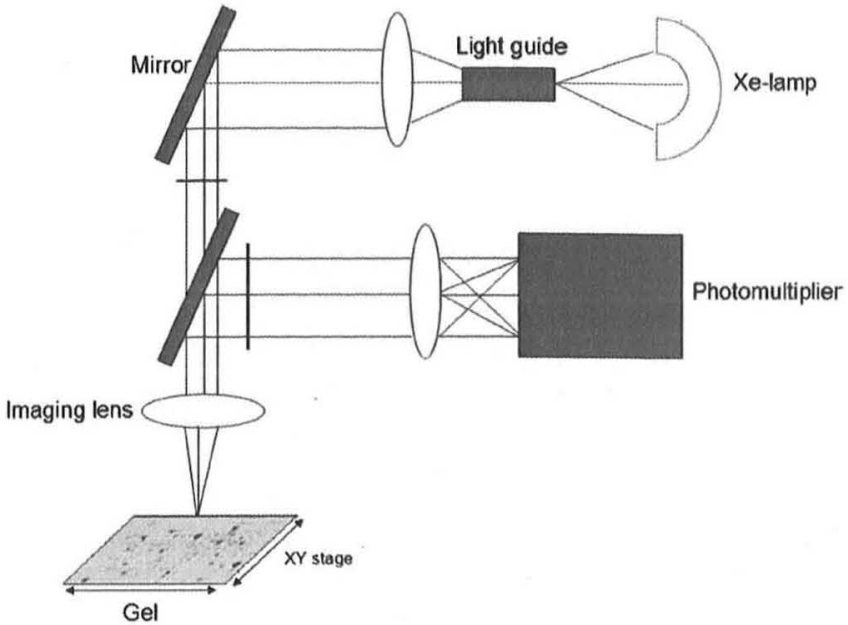

Fig. 1. Scheme of the gel bioanalyzer (LaVision-Biotec, Bielefeled, Germany), adapted after http://www.lavisionbiotec.com/en/microscopy-products/gelreader/.

lenses onto a photomultiplier detector. A UV band-pass filter $(280-400 \mathrm{~nm})$ is incorporated to block the excitation light from the detection system. From four filter positions (one for UV excitation, three for visible fluorescence), the UV filter transmitting light at $\lambda=343 \pm 65 / 2 \mathrm{~nm}$ was employed. The large reading area $\left(30 \times 35 \mathrm{~cm}^{2}\right)$ provided scanning of both $1 \mathrm{D}$ - and $2 \mathrm{D}$-gels. The instrument has a removable gel tray and is equipped to read unstained as well as stained protein gels. In the present study only scanning of unstained gels was applied. High precision polycarbonate tools for localisation and isolation of protein spots were prepared by the Department's mechanical workshop; after fixation in position on the gel tray, localisation and isolation of gel spots was carried out by moving the gel tray, with positioning and scanning of the gel controlled by the LaVision-Biotec scanning software.

\subsection{Sample preparation of porcine muscle proteins}

Muscle samples were taken from Longissimus dorsi at the position of the last rib, and kept frozen at $-80^{\circ} \mathrm{C}$ until protein extraction. Homogenization was performed manually with a glass homogenizer in lysis buffer containing $50 \mathrm{mM}$ Tris- $\mathrm{HCl}$ ( $\mathrm{pH} 7.6), 150 \mathrm{mM}$ $\mathrm{NaCl}, 1 \%(\mathrm{w} / \mathrm{v}$ ) CHAPS, $1 \%(\mathrm{v} / \mathrm{v}$ ) Triton X-100, $5 \mathrm{mM} \mathrm{NaF}, 2 \mathrm{mM}$ activated $\mathrm{Na}_{3} \mathrm{VO}_{4}$ and a protease inhibitor cocktail [18]. Following homogenization, samples were centrifuged for $20 \mathrm{~min}$ at $4 \mathrm{C}$ at $15000 \times \mathrm{g}$ to remove unextracted cellular components and highmolecular weight protein complexes and insoluble proteins, and soluble proteins were subjected to 2D-gel separation; the supernatant was removed and stored at $-80^{\circ} \mathrm{C}$ until 2D-gel separation. Protein concentrations were determined by the Bradford method [19].

\subsection{Sample preparation for bacterial proteomics}

D. phosphitoxidans was grown in 11 cultures in the presence of $10 \mathrm{mM}$ sodium phosphite or $10 \mathrm{mM}$ sodium fumarate as electron donors and $10 \mathrm{mM}$ sodium sulfate as electron acceptor, and cells were harvested in the late-exponential growth phase as previously described [20]. Briefly, phosphite-induced cells were harvested under anoxic atmosphere, washed with anoxic $10 \mathrm{mM}$ Tris- $\mathrm{HCl}$ buffer ( $\mathrm{pH} 7.2$ ) and suspended in $3 \mathrm{ml}$ soluble cytoplasmic extraction reagent containing $50 \mu \mathrm{l} / \mathrm{ml}$ of protease inhibitor cocktail (Sigma, St. Louis, USA). Cell-free extracts were prepared anoxically by passing the cells $4-5$ times dropwise through a chilled French press cell. Unopened cells and cell debris were removed 
by centrifugation at $27000 \times \mathrm{g}$ (Beckman- Optima- TL Ultracentrifuge), and soluble and membrane protein fractions of proteins obtained by ultracentrifugation at $57000 \times \mathrm{g}$. Protein contents of all preparations were determined spectrophotometrically using the bicinchonic acid method (BCA protein assay; Pierce, Rockford, USA). Soluble and membrane protein fractions were stored in $200 \mu \mathrm{l}$ aliquots for 2D-gel separations.

\subsection{Proteolytic digestion}

Spots were excised manually from the gels and subjected to ingel trypsin digestion according to Mortz et al. [8]. The excised gel pieces were washed with water for $15 \mathrm{~min}$, dehydrated for $30 \mathrm{~min}$ at $25^{\circ} \mathrm{C}$ by addition of acetonitrile (ACN): water $(3: 2, \mathrm{v} / \mathrm{v})$, and dried in a Speed Vac centrifuge $(30 \mathrm{~min})$. Stained protein spots were destained by addition of $50 \mathrm{mM} \mathrm{NH} \mathrm{NCO}_{3}(15 \mathrm{~min})$, dehy drated with $3 / 2 \mathrm{ACN} / \mathrm{Milli}-\mathrm{Q}$ water, $\mathrm{v} / \mathrm{v}(15 \mathrm{~min})$ and dried in a Speed Vac centrifuge ( $30 \mathrm{~min}$ ). No destaining steps were employed for unstained protein spots. Freshly prepared trypsin solution $\left(12.5 \mathrm{ng} / \mu \mathrm{l}\right.$ trypsin in $50 \mathrm{mM} \mathrm{NH} \mathrm{HCO}_{3}$ ) was added and incubated for $45 \mathrm{~min}$ at $4{ }^{\circ} \mathrm{C}$, then for further $12 \mathrm{~h}$ at $37^{\circ} \mathrm{C}$. After removal of supernatant, peptide extraction was performed at $25^{\circ} \mathrm{C}$ with acetonitrile: $0.1 \%$ trifluoroacetic acid (TFA) in water $(3: 2)(3 \times 60 \mathrm{~min})$. Supernatant and elution fractions were combined and lyophilized to dryness.

\subsection{Mass spectrometry}

MALDI-TOF mass spectrometric analysis was carried out with a Bruker Biflex ${ }^{\mathrm{TM}}$ linear TOF (time of flight) mass spectrometer (Bruker Daltonics, Bremen, Germany) equipped with a nitrogen UV laser $(337 \mathrm{~nm})$, a dual channel plate detector, a 26-sample SCOUT source and video system, and XMASS data system for spectra acquisition and instrument control. A saturated solution of $\alpha$ cyano-4-hydroxy-cinnamic acid (HCCA) in ACN/0.1\% trifluoroacetic acid in water $(2: 1(\mathrm{v} / \mathrm{v}))$ was used as the matrix. Aliquots of $0.8 \mu \mathrm{l}$ of the sample solution and saturated matrix solution were mixed on the stainless steel MALDI target and allowed to dry. Acquisition of spectra was carried out at an acceleration voltage of $20 \mathrm{kV}$ and a detector voltage of $1.5 \mathrm{kV}$.

Digestion mixtures determined by MALDI-MS were directly used for database search employing the MASCOT peptide mass fin- gerprinting (PMF) search engine (http://www.matrixscience.com), employing search and acceptance criteria for protein identification as follows: $0.5-1.2 \mathrm{Da}$ mass error tolerance; 2 missed cleavage sites permitted; methionine oxidation as variable modification; carbamidomethyl (cysteine) as fixed modification. Other Proteobacteria was used as taxonomy for the bacterial proteins (277,231 sequences) and Mammalia as taxonomy for all other investigated proteins ( 484,669 sequences), with a minimum number of three matched peptides. The database employed was NCBInr 20060712 (3,783,042 sequence entries, 1,304,471,729 residues), a compilation of several databases including SWISS-PROT, PIR, PRF, PDB and GenBank CDS translations.

\section{Results and discussion}

\subsection{Protein detection by stain-free visualization using native fluorescence}

SDS-PAGE separations of several model proteins were initially tested by comparison of Colloidal Coomassie, silver staining, and stain-free detection using native fluorescence and fluorescence upon fixation with halogenated derivatives. It has been previously shown that the incorporation of halogenated compounds in polyacrylamide gels either prior to polymerization [11] or subsequent to the electrophoretic separation [14], followed by UV illumination provide fluorescent protein derivatives. However, our model studies showed that protein fixation using halogenated derivatives after SDS-PAGE can be omitted, as illustrated by SDS-PAGE separations of lysozyme and myoglobin without fixation (Fig. 2a), and with fixation (30 min) in 12\% trichloroacetic acid (TCA) (Fig. 2b). Upon scanning with the gel bioanalyzer, protein bands were detected with and without fixation, however bands with approximately $30-50 \%$ higher abundance were observed without protein fixation for the model proteins studied (Fig. 2). A further increased abundance was obtained by washing the gel band with water which leads to reduced background fluorescence; however, a washing step was found to cause decreased stability of the protein fluorescence. We observed an increased stability (slower decrease) of the fluorescence intensity in proteins within $48 \mathrm{~h}$ after fixation in $12 \%$ TCA (data not shown), which may be explained by a more stable fluorescence emission of UV-reaction products of tryptophane with TCA [14].

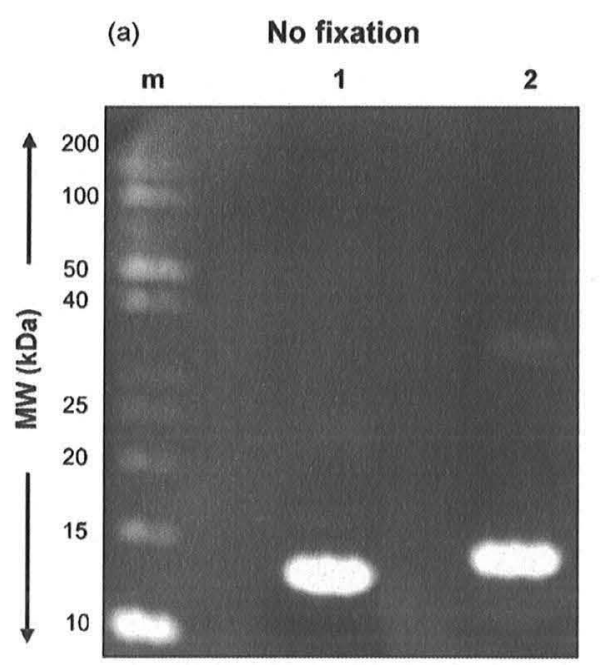

Fig. 2. Comparison of native fluorescence detection for $12 \%$ SDS-PAGE separation of $5 \mu \mathrm{g}$ hen eggwhile lysozyme (lane 1 ) and $5 \mu \mathrm{g}$ myoglobin (lane 2 ) with and without fixation with trichloroacetic acid. For each gel a $5 \mu \mathrm{l}$ aliquot of molecular weight marker (Fermentas; $10-200 \mathrm{kDa}$ ) was used (lane m). (a) No protein fixation was performed; (b) proteins were fixed for $30 \mathrm{~min}$ with a $12 \%$ aqueous trichloroacetic acid solution. 


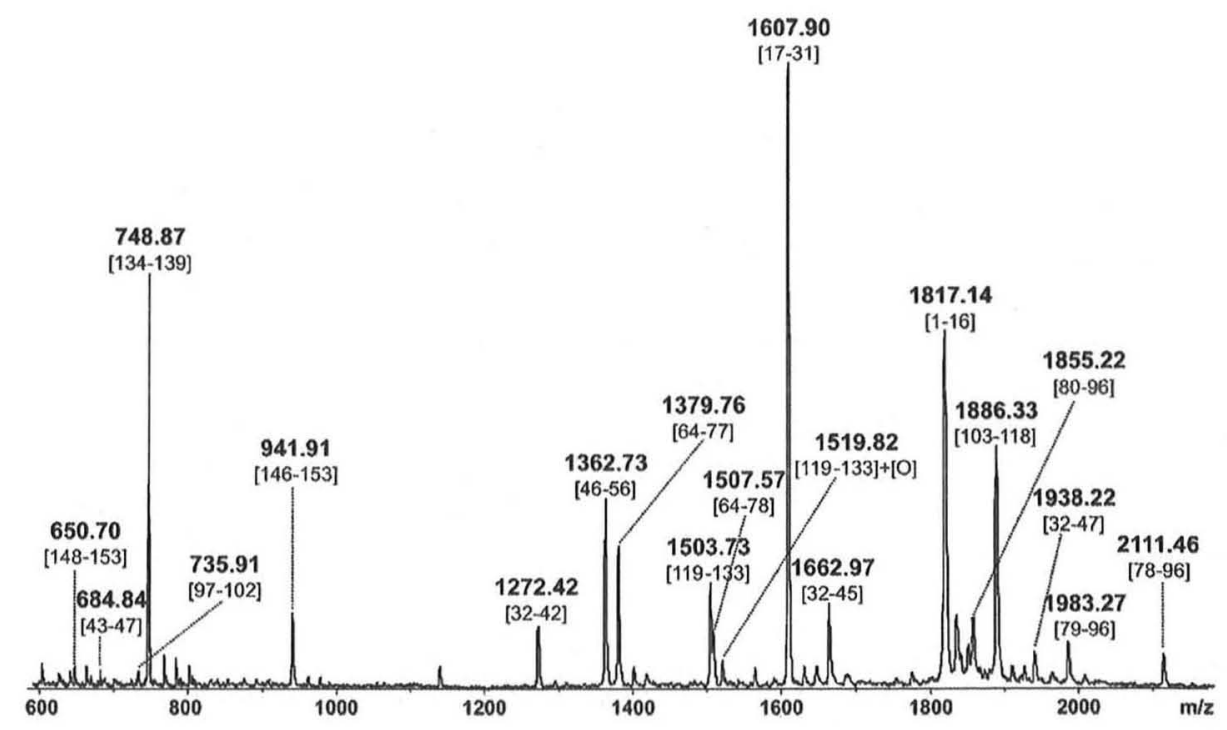

Fig. 3. MALDI-TOF mass spectrum of horse heart myoglobin (lane 2 in Fig. 2) identified after stain-free gel detection and in-gel digestion with trypsin.

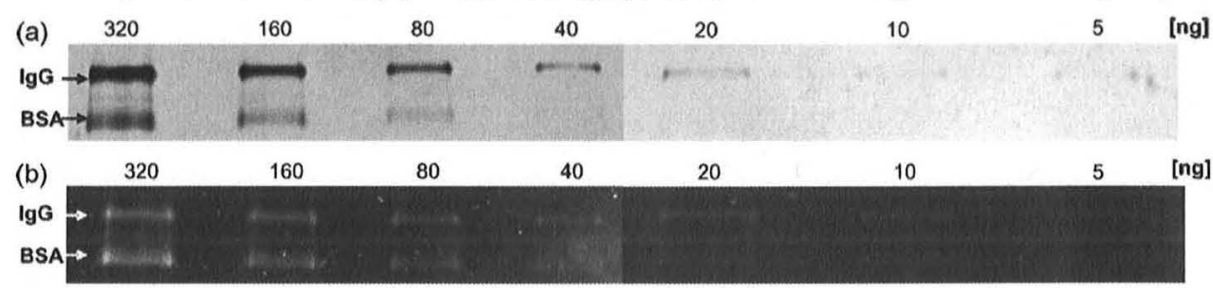

Fig. 4. Sensitivity of stain-free fluorescence detection and visualization in comparison with silver visualization. Protein samples, IgG (150 kDa heavy and light chain dimer) and BSA ( $67 \mathrm{kDa}$ ) were separated in 7 lanes at $320-5 \mathrm{ng}$, Gel areas presented are zoomed regions from $12 \%$ SDS-PAGE separations. (a) Silver stained gels; (b) native fluorescence gels.
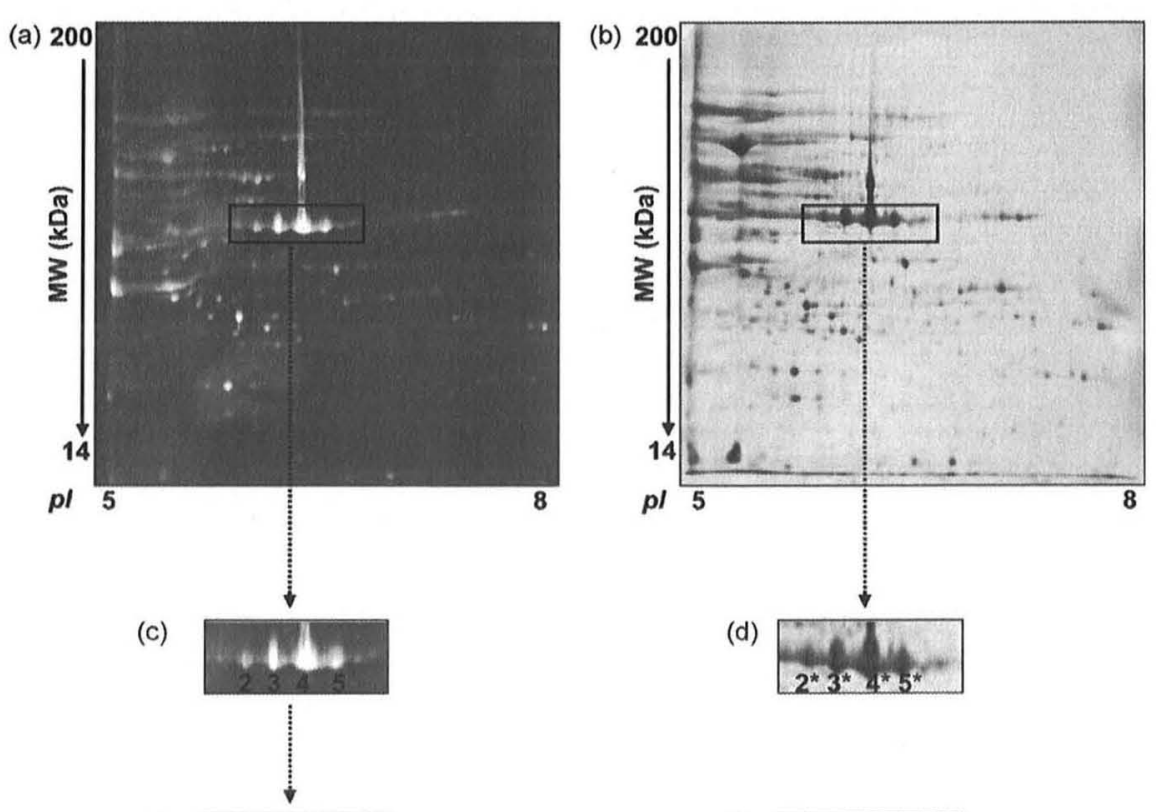

(e)

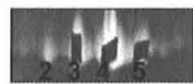

Coomassie staining

(f)

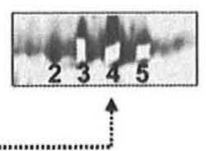

Fig. 5. Detection and localisation of protein spots in 2D-gels by native fluorescence using precise spot picking tools. Separation gels were prepared with protein extracts from Desulfotignum phosphitoxidans (grown with phosphite/fumarate/sulfate; approximately $0.4 \mathrm{mg}$ total protein were applied onto $17 \mathrm{~cm}$ IPG strips $5-8$ ) [20]. Spot picking tools were used to cut spots from unstained gels. (a) Unstained gel with native fluorescence detection. (b) 2D-gel stained with Coomassie, prepared in identical manner with the bioanalyzer gel. (c) Zoomed area from gel a. (d) Zoomed area from gel b. (e) Region as in c, after excision of gel spots. (f) Gel area from (e) after Coomassie staining. The numbers 2-5 denote the spots from the unstained gel with native fluorescence detection. Following excision of the spots the gel was stained with Coomassie, and numbers $2^{*}, 3^{*}, 4^{*}$ and $5^{*}$ denote the spots from the Coomassie-stained gel. Spots 3 and 4 from the native fluorescence gels were used for protein identification. 
The MALDI-mass spectrometric identification of the gel band of myoglobin isolated from the gel presented in Fig. 2a (lane 2, without fixation) is shown in Fig. 3 (see details of protein localisation and isolation below). The band was excised and in-gel digested with trypsin, and the digest mixture analysed by MALDI-TOFMS and used for database search employing the MASCOT peptide mass fingerprinting (PMF) search engine. The database search provided unequivocal identification of myoglobin (Fig. 3). For the gel scanned with the bioanalyzer, no destaining step was required, which provided high-sensitivity and considerably lower sample preparation time compared to staining procedures. For the $320 \mathrm{ng}$ protein band, identification was obtained with a score of $83(64 \%$ sequence coverage). Thus, these model studies clearly showed that gel separation of proteins detected by native fluorescence without a fixation step represents an efficient and sensitive approach for mass spectrometric identification, provided sufficiently fast

(a)

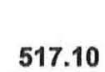

[294-297]

1146.37

[249-258] 1596.81

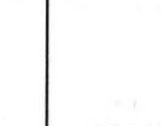

$[187-197]$

690.78

[23-28]
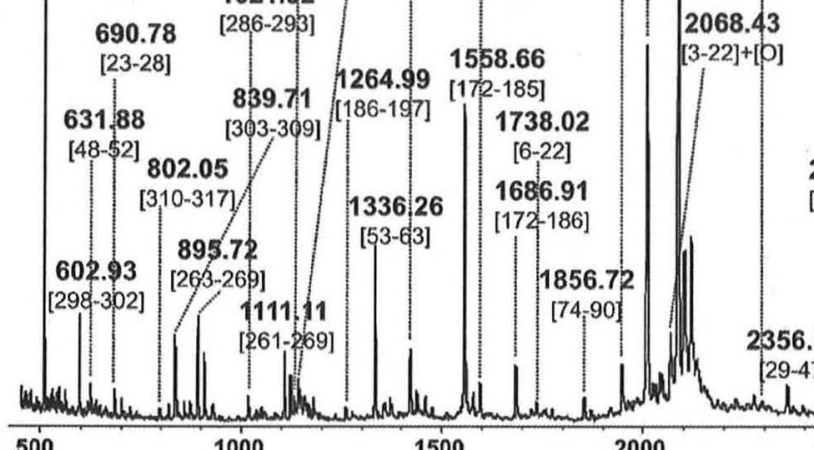

(14)

[261-274]

[48-52]

839.71 $1264.99 \begin{array}{r}1558.66 \\ {[172-185]}\end{array}$

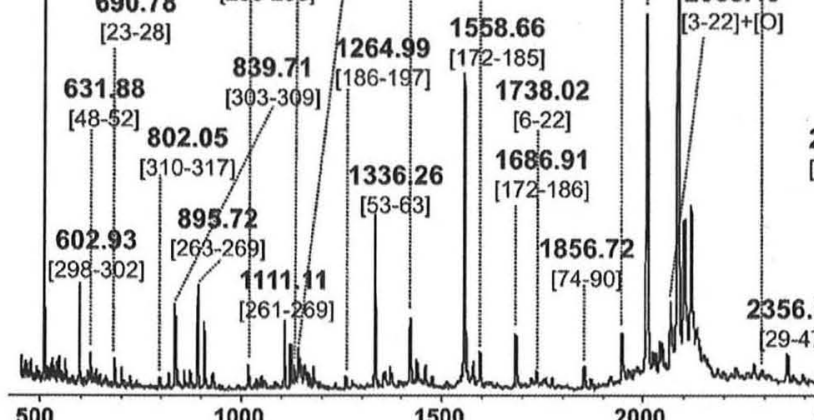

[152-171]

(b)

2009.58

[152-169]

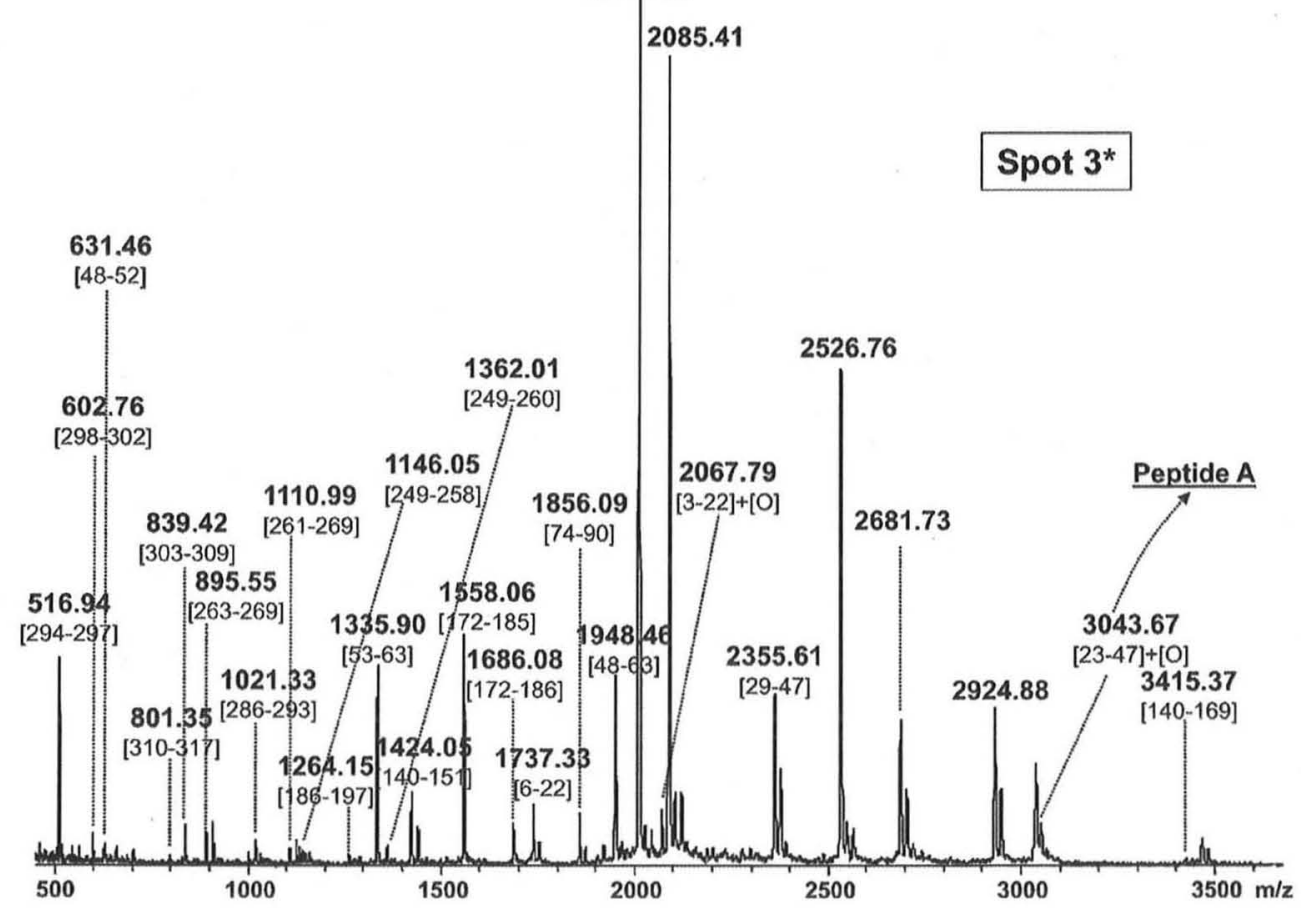

Fig. 6. MALDI-TOF mass spectrometric identification of NAD(P)-dependent epimerase/dehydratase from Desulfotignum phosphitoxidans. Spots 3 (Fig. 5c) and $3 *$ (Fig. 5d) were excised from the native fluorescence gel and from the Coomassie-stained gel, digested with trypsin and analysed by MS. Masses of peptide ions for protein spots 3 (Fig. 6 a) and $3^{*}$ (Fig. 6b) were compared with the calculated masses for fragment ions of the open reading frame obtained from genomic DNA amplification. Masses corresponding to peptides $A$ and $B$ (see Table 1) which were previously found to be phosphorylated using metal ion affinity enrichment (IMAC) [20], are observed in Fig. 6a (peptide B) and $b$ (peptide A caring one oxidation) 
mass spectrometric analysis of the gel separated proteins is performed.

\subsection{Detection sensitivity of proteins by stain-free native florescence in comparison with staining procedures}

The sensitivity of the stain-free native fluorescence detection with the gel bioanalyzer was evaluated in comparison with standard staining procedures by Coomassie and silver visualization. Corresponding gel separations of mixtures of the two model proteins, immunoglobulin- $G$ and bovine serum albumin at concentrations of $320-5 \mathrm{ng} /$ band are compared in Fig. 4. These results indicated comparable sensitivities for the UV fluorescence detection and silver staining (Fig. $4 a$ and $b$ ) with detection limits of approximately $1-5 \mathrm{ng}$ for IgG, while the corresponding Coomassiestained gel band was not detectable at this concentration (data not shown). The detection limit in the low nanogram range observed is in good agreement with sensitivity data reported by Roegener et al. [9].

\subsection{Localisation of proteins in stain-free gel separations for mass spectrometric proteome analysis}

In order to evaluate the isolation and localisation of protein bands using the stain-free detection, protein extracts from the bacterium $D$. phosphitoxidans were separated and analysed by $2 \mathrm{D}-$ gel electrophoresis. Corresponding $10 \%$ separation gels of bacterial extract were prepared and visualized by native fluorescence and Coomassie staining (Fig. 5a and b). Using the Bio-Rad PDQuest software approximately 340 proteins were detected in the 2D-gel with native fluorescence (Fig. 5a), while the detection of approximately 350 protein spots was estimated in the Coomassie-stained gel (Fig. 5b), suggesting a comparable detection sensitivity with native fluorescence and Coomassie staining by the number of protein spots. Since the fluorescence signal depends on the abundance of aromatic amino acids in the protein, proteins with low abundance in aromatic amino acid residues may not be visualized using this method.

Proteins from $D$. phosphitoxidans, specifically induced in the presence of phosphite have been identified in a previous study as a NAD(P)-dependent epimerase/dehydratase (accession number of gene, ABU54327) [20]. This protein from the bacterium with a cur-

\section{Table 1}

Protein identifications in proteome applications using native fluorescence for visualization of gel separated proteins. Protein spots were excised from 2D-gels (unstained gels used for native florescence) and in-gel digested with trypsin. Digest mixtures were measured by MALDI-TOF-MS and the resulted masses were used for database search employing MASCOT PMF search engine. For Desulfotignum phosphitoxidans samples masses of peptide ions for each protein spot (spots 3, 4 from Fig. 5) were compared with the calculated masses for fragment ions of the open reading frame obtained from genomic DNA amplification. Spots 3 and 4 led to the identification of the same protein but with different phosphorylation degrees. For spots 6 and 7 see Fig. 7a.

\begin{tabular}{|c|c|c|c|c|c|}
\hline Spots & Identified protein & Acc. no. ${ }^{a}$ & $\begin{array}{l}\text { Phosohorylated } \\
\text { peptide/Pb }\end{array}$ & Score & Seq. cov. \\
\hline 3 & $\begin{array}{l}\mathrm{NAD}(\mathrm{P}) \text {-dependent } \\
\text { epimerase/dehydratase }^{\mathrm{c}}\end{array}$ & ABU54327 & $A^{d} / 1 ; B^{e} / 4$ & - & $98.7 \%$ \\
\hline 4 & $\begin{array}{l}\mathrm{NAD}(\mathrm{P}) \text {-dependent } \\
\text { epimerase/dehydratase }^{c}\end{array}$ & ABU54327 & $\mathrm{B} / 4$ & - & $97.8 \%$ \\
\hline 6 & Skeletal alpha-actin ${ }^{f}$ & P68137 & - & 92 & $70.2 \%$ \\
\hline 7 & Creatine kinase $M$ chain ${ }^{f}$ & Q5XLD3 & - & 78 & $90.1 \%$ \\
\hline
\end{tabular}

a Accession numbers are from SWISS-PROT or TrEMBL database. For spots 3, 4 and

$3^{*}, 4^{*}$ the accession number is from GeneBank database.

b Number of phosphorylations found.

c Desulfotignum phosphitoxidans.

d Peptide A: ${ }^{23}$ LIEMGKDVTLFDNNEOHNMYEETOK ${ }^{47}$

e Peptide B: ${ }^{91}$ FKOEVETVEVNVIGTLNVLOASLDTGVK ${ }^{118}$

f Sus scrofa. rently unknown genome has been elucidated by a combination of inverted-PCR based on $\mathrm{N}$-terminal sequences providing possible open reading frame candidates, and high resolution mass spectrometric identification of proteolytic peptide masses. Protein spots corresponding to different phosphorylated isoforms were excised from the gels for mass spectrometric analysis (spots $2-5$; Fig. $5 c$; spots $2^{*}, 3^{*}, 4^{*}, 5^{*}$; Fig. $5 d$ ). The correct localisation of the gel spots on the native fluorescence gel was ascertained by excision

(a)

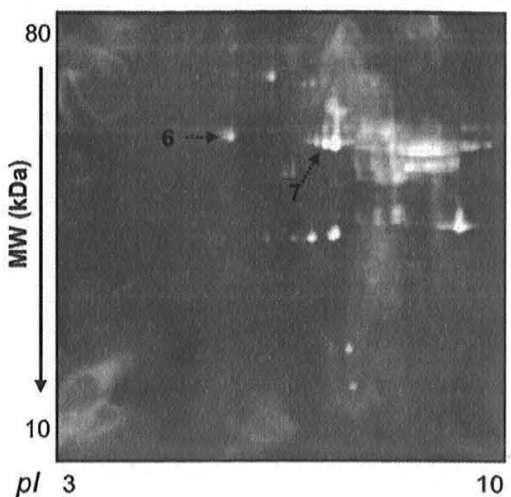

(b)
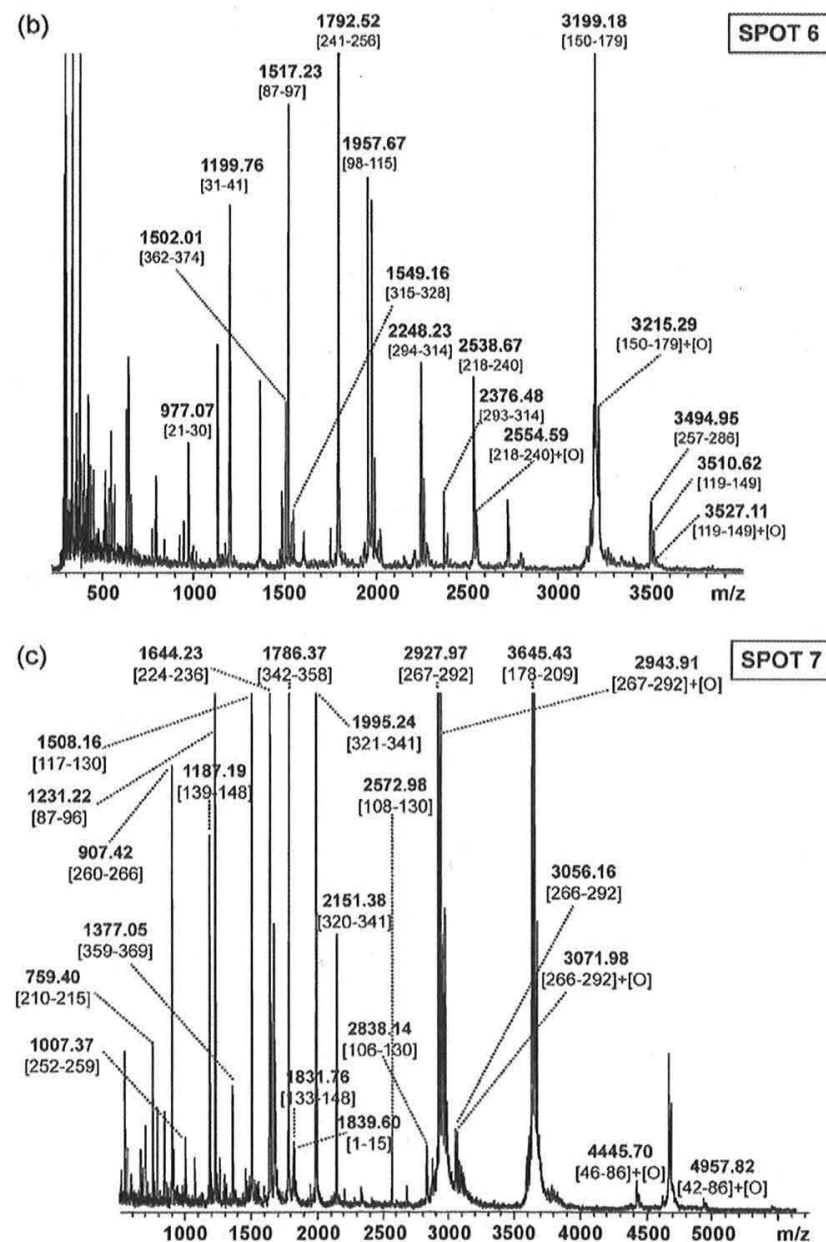

Fig. 7. Protein identification after 2D-gel separation (12.5\% SDS-PAGE) of a postmortem porcine muscle sample ( $1.5 \mathrm{mg}$ total protein per gel). (a) Gel visualized by native fluorescence. Spots 6 and 7 were excised and in-gel digested with trypsin. (b) MALDI-TOF mass spectrum of the digestion mixture of spot 6 (labeled peaks correspond to the identified peptides from porcine skeletal alpha-actin).(c) Identification of spot 7, porcine creatine kinase M (with identified peptides labeled) 
of the protein spot, Coomassie staining and comparison with the position of the Coomassie-stained spots (Fig. 5d-f). The mass spectrometric identification of spot 3 from the native fluorescence gel as a penta-phosphorylated NAD(P)-epimerase/dehydratase [20] is shown in Fig. 6a, in agreement with the phosphorylated protein identified from the Coomassie-stained gel. Identifications of different phosphorylated isoforms are illustrated in Table 1. The mass spectrometric identification of these isoforms ascertained the precise and reproducible localisation of fluorescence-visualized gel spots with the native fluorescence gel bioanalyzer. Fig. 6b illustrates the MALDI-TOF-MS identification of the same protein (NAD(P)dependent epimerase/dehydratase) from spot $3^{*}$ excised from the Coomassie-stained gel. A total of 21 tryptic peptide fragments were found to be common for both MALDI-TOF spectra of spots 3 (Fig. 6a) and 3* (Fig. 6b).

\subsection{Application of stain-free gel separation to mass spectrometric proteome analysis of porcine muscle tissue}

Examples of mass spectrometric proteome analyses using the stain-free gel bioanalyzer to porcine muscle skeleton proteins isolated post-mortem are summarised in Fig. $7(a-c)$. The rate and extent of post-mortem metabolic processes of skeleton muscle proteins have recently found increasing interest, and it is generally believed that changes such as degradation and oxidation post-mortem may influence the meat properties [21]; e.g., tenderization processes have been associated with calpains and their inhibitors, calpastatins, and proteins involved in carbonylation may be potentially useful oxidation biomarkers [22-24]. Following tryptic digestion of isolated gel spots, the MALDI-MS analysis provided the identification of two proteins, alpha-actin and creatine kinase $\mathrm{M}$, both of which showed modifications by oxidation (Table 1 ; Fig. 7b and c) [25].

In applications to porcine muscle proteins, high sensitivities for alpha-actin and creatine kinase $\mathrm{M}$ were observed using native fluorescence detection, which can be explained by the amino acid compositions of these proteins with a high content of aromatic residues. Sequences of alpha-actin and creatine kinase $M$ are shown in Figs. 1 and 2 of the Supplementary Material. Native fluorescence emission of protein is mainly due to tryptophane residues, with some contribution of tyrosine and phenylalanine (alpha-actin, 4 $\mathrm{W} ; 16 \mathrm{Y} ; 12 \mathrm{~F}$; creatine kinase M, $4 \mathrm{~W} ; 10 \mathrm{Y} ; 16 \mathrm{~F}$ ).

\section{Conclusions}

In this study we present stain-free detection and visualization of proteins in gels using native fluorescence as an efficient and sensitive approach for mass spectrometric proteome analysis. Using the stain-free gel bioanalyzer enabled the detection and mass spectrometric identification of proteins from gel spots at detection limits in the low nanogram range, comparable to silver staining. Moreover, this approach does not require any post-electrophoretic manipulation by destaining and fixation, thus providing advantages for mass spectrometric analysis by reduced background and time needed for sample preparation. The use of fluorescence detection with 2Dgel electrophoresis suggests that this technique can be developed for automated, high-throughput technologies of proteome analysis. Thus, the stain-free fluorescence visualization should be a useful complement to staining techniques of gel electrophoresis for mass spectrometric protein analysis.

\section{Acknowledgments}

We are grateful to Michael Gross for our long-standing collaboration, his advise and support in biopolymer mass spectrometry. We thank Martin Schütte and Bernd Müller-Zülow, LaVision-BioTec for technical support regarding the gel bioanalyzer. This work has been supported by the Deutsche Forschungsgemeinschaft, Bonn, Germany (FOR-753), and the University of Konstanz (Proteostasis Research Center).

\section{References}

[1] N. Dyballa, S. Metzger, Fast and sensitive colloidal Coomassie G-250 staining for proteins in polyacrylamide gels, J. Vis. Exp. 30 (2009)

[2] H.W. Ott, A. Griesmacher, M. Schnapka-Koepf, G. Golderer, A. Sieberer, M. Spannagl, B. Scheibe, S. Perkhofer, K. Will, U. Budde, Analysis of von Willebrand factor multimers by simultaneous high- and low-resolution vertical SDS-agarose gel electrophoresis and Cy5-labeled antibody high-sensitivity fluorescence detection, Am. J. Clin. Pathol. 133 (2010) 322-330.

[3] R.J. Simpson, SYPRO Orange Fluorescent Staining of Protein Gels, Cold Spring Harb Protoc. 2010 pdb prot5414.

[4] R.J. Simpson, Rapid Coomassie Blue Staining of Protein Gels, Cold Spring Harb Protoc. 2010 pdb prot5413.

[5] V. Neuhoff, N. Arold, D. Taube, W. Ehrhardt, Improved staining of proteins in polyacrylamide gels including isoelectric focusing gels with clear background at nanogram sensitivity using Coomassie Brilliant Blue G-250 and R-250, Electrophoresis 9 (1988) 255-262.

[6] J. Heukeshoven, R. Dernick, Improved silver staining procedure for fast staining in PhastSystem Development Unit. I. Staining of sodium dodecyl sulfate gels, Electrophoresis 9 (1988) 28-32.

[7] J.F. Lin, Q.X. Chen, H.Y. Tian, X. Gao, M.L. Yu, G.J. Xu, F.K. Zhao, Stain efficiency and MALDI-TOF MS compatibility of seven visible staining procedures, Anal. Bioanal. Chem. 390 (2008) 1765-1773.

[8] E. Mortz, O. Vorm, M. Mann, P. Roepstorff, Identification of proteins in polyacrylamide gels by mass spectrometric peptide mapping combined with database search, Biol. Mass Spectrom. 23 (1994) 249-261.

[9] J. Roegener, P. Lutter, R. Reinhardt, M. Bluggel, H.E. Meyer, D. Anselmetti, Ultrasensitive detection of unstained proteins in acrylamide gels by native UV fluorescence, Anal. Chem, 75 (2003) 157-159

[10] H. Yamamoto, M. Nakatani, K. Shinya, B.H. Kim, T, Kakuno, Ultraviolet imaging densitometry of unstained gels for two-dimensional electrophoresis, Anal. Biochem. 191 (1990) 58-64.

[11] C.L. Ladner, J. Yang, R.J. Turner, R.A. Edwards, Visible fluorescent detection of proteins in polyacrylamide gels without staining, Anal. Biochem. 326 (2004) 13-20.

[12] C. Sluszny, E.S. Yeung, One- and two-dimensional miniaturized electrophoresis of proteins with native fluorescence detection, Anal. Chem. 76 (2004) 1359-1365

[13] Z. Zhao, Y. Aliwarga, M.D. Willcox, Intrinsic protein fluorescence interferes with detection of tear glycoproteins in SDS-polyacrylamide gels using extrinsic fluorescent dyes, J. Biomol. Technol. 18 (2007) 331-335.

[14] D. Kazmin, R.A. Edwards, R.J. Turner, E. Larson, J. Starkey, Visualization of proteins in acrylamide gels using ultraviolet illumination, Anal. Biochem. 301 (2002) 91-96

[15] http://www.lavisionbiotec.com/en/microscopy-products/gelreader/.

[16] B. Schink, M. Friedrich, Phosphite oxidation by sulphate reduction, Nature 406 (2000) 37.

[17] B. Schink, V. Thiemann, H. Laue, M.W. Friedrich, Desulfotignum phosphitoxidans sp. nov., a new marine sulfate reducer that oxidizes phosphite to phosphate, Arch. Microbiol. 177 (2002) 381-391.

[18] J. Lee, N. Valkova, M.P. White, D. Kültz, Proteomic identification of processes and pathways characteristic of osmoregulatory tissues in spiny dogfish shark (Squalus acanthias), Comp. Biochem. Physiol. Part D1 (2006) 328-343.

[19] M.M. Bradford, A rapid and sensitive method for the quantitation of microgram quantities of protein utilizing the principle of protein-dye binding, Anal. Biochem. 72 (1976) 248-254.

[20] D.D. Simeonova, I. Susnea, A. Moise, B. Schink, M. Przybylski, "Unknown genome" proteomics: a new NADP-dependent epimerase/dehydratase revealed by $\mathrm{N}$-terminal sequencing, inverted $\mathrm{PCR}$, and high resolution mass spectrometry, Mol. Cell Proteomics 8 (2009) 122-131.

[21] M. Koohmaraie, Biochemical factors regulating the toughening and tenderization processes of meat, Meat Sci. 43 (1996) 193-201.

[22] J. Huang, N.E. Forsberg, Role of calpain in skeletal-muscle protein degradation, Proc. Natl. Acad. Sci. U.S.A. 95 (1998) 12100-12105.

[23] M.E. Doumit, M. Koohmaraie, Immunoblot analysis of calpastatin degradation: evidence for cleavage by calpain in postmortem muscle, J. Anim. Sci. 77 (1999) $1467-1473$.

[24] R. Lametsch, P. Roepstorff, E. Bendixen, Identification of protein degradation during post-mortem storage of pig meat, J. Agric. Food Chem. 50 (2002) $5508-5512$.

[25] B. Bernevic, D. Galetskiy, A. Petre, C. Werner, M. Wicke, K. Schellander, M. Przybylski, Porcine muscle protein degradation and oxidation post-mortem revealed by high resolution mass spectrometric proteome analysis, Abstr., Third Int. Symposium Oxidative Post-translational Modifications, Boston, 2008. 